\title{
Control of (pre)-analytical aspects in immunoassay measurements of metabolic hormones in rodents
}

\author{
Maximilian Bielohuby', Martin Bidlingmaier ${ }^{2}$ and Uwe Schwahnn ${ }^{1}$ \\ ${ }^{1}$ Sanofi-Aventis Deutschland GmbH, R\&D, Industriepark Höchst, Frankfurt, Germany \\ ${ }^{2}$ Endocrine Research Laboratories, Medizinische Klinik und Poliklinik IV, Klinikum der Universität München, Munich, Germany \\ Correspondence should be addressed to M Bielohuby: Maximilian.Bielohuby@sanofi.com
}

\begin{abstract}
The measurement of circulating hormones by immunoassay remains a cornerstone in preclinical endocrine research. For scientists conducting and interpreting immunoassay measurements of rodent samples, the paramount aim usually is to obtain reliable and meaningful measurement data in order to draw conclusions on biological processes. However, the biological variability between samples is not the only variable affecting the readout of an immunoassay measurement and a considerable amount of unwanted or unintended variability can be quickly introduced during the pre-analytical and analytical phase. This review aims to increase the awareness for the factors 'pre-analytical' and 'analytical' variability particularly in the context of immunoassay measurement of circulating metabolic hormones in rodent samples. In addition, guidance is provided how to gain control over these variables and how to avoid common pitfalls associated with sample collection, processing, storage and measurement. Furthermore, recommendations are given on how to perform a basic validation of novel single and multiplex immunoassays for the measurement of metabolic hormones in rodents. Finally, practical examples from immunoassay measurements of plasma insulin in mice address the factors 'sampling site and inhalation anesthesia' as frequent sources of introducing an unwanted variability during the preanalytical phase. The knowledge about the influence of both types of variability on the immunoassay measurement of circulating hormones as well as strategies to control these variables are crucial, on the one hand, for planning and realization of metabolic rodent studies and, on the other hand, for the generation and interpretation of meaningful immunoassay data from rodent samples.
\end{abstract}

\author{
Key Words \\ - variability \\ - mice \\ - immunoassay \\ - ELISA \\ - insulin \\ - plasma hormones \\ - assay performance \\ - assay validation \\ - multiplex \\ - metabolism \\ - endocrine factors
}

\section{Introduction}

After the discovery, in 1959, of the radioimmunoassay which enabled the measurement of insulin in human plasma (1), circulating metabolic hormones such as insulin (2) or growth hormone $(3,4)$ were quickly investigated for research purposes in laboratory rodents. The replacement of radioactivity by an enzymatic label in 1971 by Engvall and Perlmann (ELISA) (5) as well as by van Weemen and Schuurs (EIA) (6), and the associated increased handling convenience led to a boost in using immunoassays and ever since they have arrived as standard tools in science. Today, the analysis of metabolic hormones by immunoassay is an essential diagnostic tool in medicine. In parallel, immunoassay measurements of circulating parameters have been crucial http://www.endocrineconnections.org https://doi.org/10.1530/EC-18-0035 (c) 2018 The authors Published by Bioscientifica Ltd

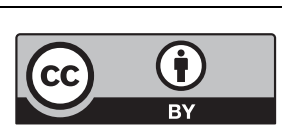

This work is licensed under a Creative Commons Attribution 4.0 International License. 
for an overwhelming number of scientific discoveries in metabolic diseases. When measuring hormones in laboratory rodents, the paramount aim usually is to generate scientific data rather than to acquire diagnostic information. Especially after the identification of a novel circulating hormone or after the recognition of novel functions of a previously known parameter, the interest to measure this specific parameter in the circulation of rodents may arise or may experience a revival among a broader scientific community. Immunoassays are particularly suitable to serve this demand for a number of reasons. If appropriate antibodies reliably recognizing the antigen of interest are available, larger batches of immunoassays are relatively easy to produce in a short amount of time. In addition, the technical procedures to conduct immunoassays are established in most research labs and many groups have access to the required analytical equipment. It is important to distinguish between immunoassays installed for diagnostic purposes in humans and immunoassays used to detect circulating metabolic parameters in rodents for research purposes. The immunoassay classification 'for research purposes only' allows assay manufacturers to quickly provide the necessary tools to the research community without the need for exhaustive assay validation processes, which are typically required for diagnostic assays in human medicine. At the same time, the lack of thorough validation processes for novel immunoassays due to the need for timely solutions also comes with an important limitation: the quality of 'research immunoassays' is often worse compared to immunoassays used in diagnostic laboratory medicine. This topic has also recently been highlighted in an editorial discussing immunoassays for the measurement of (rodent) steroid hormones (7). It has perspicaciously been warned that using commercially available rodent immunoassays without implementing the traditional controls, such as spiked samples, mixing experiments and serial dilutions can seriously impact the quality of research data. The author also raised the awareness that self-generated data from specific samples used in the experiments should be used to describe the performance of an immunoassay rather than reporting performance characteristics provided by the manufacturer in the 'instructions for use' (IFU) (7).

The primary aim of this review is to bring this issue to the attention of researches with the goal to also provide some guidance on how to avoid common pitfalls. Furthermore, recommendations are given how to perform a basic validation of novel immunoassays for measurement of metabolic hormones in rodents. Finally, practical measurement examples should increase the awareness for the potential introduction of unwanted variability during the pre-analytical and the analytical phases.

\section{Sources of variability in immunoassays}

By nature, every analytical method is subject to variability. In addition to the biological variability usually being the desired readout of immunoassay analyses in research, pre-analytical and analytical variability also affect the measurement readout in immunoassays. Preanalytical and analytical variability in this context relate to the phenomenon that measurement readouts in immunoassay always need to be regarded as relative analyte concentrations (mostly relative to a calibrator curve) and thus the term 'variability' seeks to describe the difference between the true analyte concentration in the sample and the observed measurement result $(8,9)$. Both types of variability are typically less well controlled and studied in research immunoassays used for measurement of metabolic hormones in rodents compared to established immunoassays used in routine laboratory diagnostics. Pre-analytical variability describes the variability potentially introduced by differences related to sample collection, sample preparation and processing as well as sample storage $(9,10,11,12,13)$. In contrast, analytical variability summarizes the variability introduced into a measurement result by factors that are directly connected to the analytical method, such as the choice and specificity of antibodies, assay buffers, assay design (sandwich vs competitive), matrix differences between calibrators and samples, incubation times or interference with other endogenous ligands $(8,9,14,15)$. Pre-analytical variability sometimes is regarded to be of minor importance. However, it has been estimated that the variability introduced during this phase accounts for up to $93 \%$ of the total errors encountered within the entire diagnostic process in human laboratory medicine (12). Considering that the pre-analytical phase in human medicine - despite well-known shortcomings already has a high degree of standardization, it must be assumed that in the less well-controlled and standardized environment of many rodent studies the factor 'preanalytical variability' might even more severely affect sample quality.

$$
\text { http://www.endocrineconnections.org }
$$
https://doi.org/10.1530/EC-18-0035
(C) 2018 The authors Published by Bioscientifica Ltd

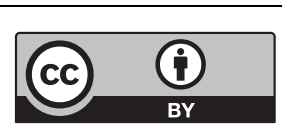

This work is licensed under a Creative Commons Attribution 4.0 International License. 


\section{Blood sampling site and effect of isoflurane anesthesia}

One aspect that is relatively well known and described within the perimeter 'pre-analytical and unwanted biological variability' is that - depending on the analyte - a considerable amount of variability can potentially be introduced by the choice of the blood sampling method and site. Most available data and recommendations have been reported for humans and domestic animals (e.g. (16, $17,18,19,20)$ ), with significantly less data reported for rodents, particularly with respect to potential effects on circulating endocrine parameters. Not surprisingly, also in rodents the blood sampling site can affect measurement results. In this context, a solid amount of data has been reported for rodents showing that the blood sampling site and technique has a significant impact upon the measurement of many clinical chemistry parameters including hematopoietic cells, plasma glucose, liver lipids and enzymes and the presence of clots or hemolysis (21, $22,23)$. For example, Hoggatt et al. have demonstrated in mice that sampling from central sites yields lower numbers of red and white blood cells compared with blood sampled peripherally (23). Schnell and colleagues also showed in mice that the site of blood collection plays a substantial role for measurement of many clinical chemistry parameters such as transaminases or lipases (24). Similarly, Fernández et al. found significant differences in 8 out of the 9 biochemical parameters studied (including transaminases, albumin, triglycerides, total cholesterol and creatinine) between submandibular vein and retrobulbar plexus blood collection in C57BL/6J mice (25). This type of systematic analysis is less often conducted in rodent studies and data are usually only reported for individual hormones. Among the reported hormones, particular attention has been given to hormones belonging to the hypothalamic-pituitary-adrenal (HPA) axis as well as to adrenal medullary hormones $(26,27,28,29,30)$.

As another example of a frequently measured metabolic parameter in rodents, we here depict that the sampling site can affect the measurement results of plasma insulin in mice. Insulin concentrations were measured in samples collected either via puncture of the tail vein or from the retrobulbar sinus of adult C57BL/6J mice. During the whole sampling process, mice remained under isoflurane narcosis and the two blood samples were collected within 3 min per mouse into prefilled EDTA tubes on ice. Further details on sampling and analytical procedures can be found in the 'Materials and methods' section. In another cohort of 20 mice, we studied the

http://www.endocrineconnections.or https://doi.org/10.1530/EC-18-0035

2018 The author Published by Bioscientifica Ltd effect of isoflurane narcosis on plasma insulin. Therefore, two blood samples were collected from the tail vein of the same mouse, one time under isoflurane narcosis in an inhalation chamber or for the subsequent sampling without anesthesia in conscious mice. Figure $1 \mathrm{~A}$ shows that the measured plasma insulin concentrations are clearly lower in all five mice when blood was collected from the retrobulbar sinus. In contrast (and as expected), repeated sampling from the same sampling site within a short time frame did not significantly affect the measured analyte concentration (Fig. 1B). This small set of data does not claim to be a representative measure for the effect of the sampling site on plasma insulin measurements. Instead, it is intended to illustrate how quickly the alternation of the sampling site can lead to a high degree of unwanted variability in measured analyte concentrations in rodents. Thus, to avoid a potential bias in measurement results of rodent metabolic hormones within one experiment, it is very important to stay consistent with the blood sampling site, especially when comparing two or more experimental groups such as 'compound treatment' vs 'vehicle treatment' or when comparing measurement results from repeated blood samplings. Furthermore, the procedure of blood sampling should be precisely documented to
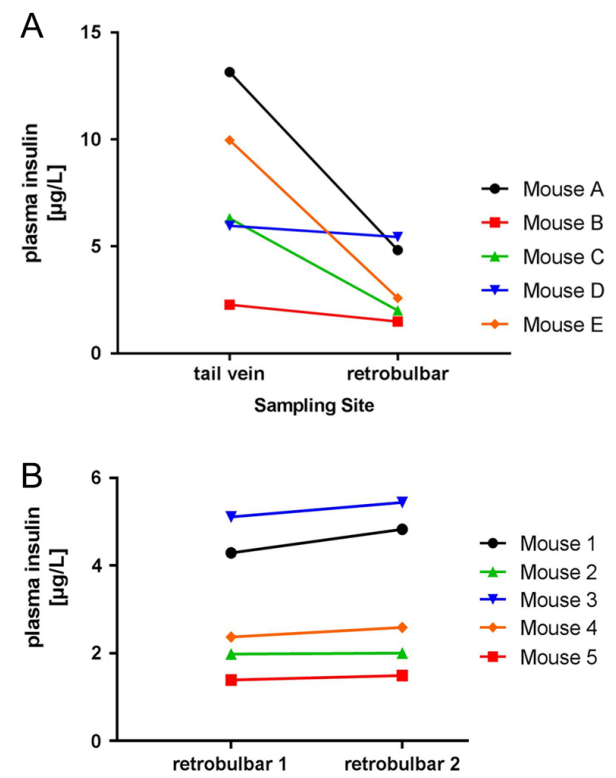

Figure 1

Example illustrating the effect of alternating the blood sampling site within short intervals (tail vein puncture vs retrobulbar sinus) on measured plasma insulin concentrations in five anesthetized C57BL/6 mice (A). Blood sampling sites were alternated from mouse to mouse to avoid the introduction of a systematic error. (B) Shows the expected absence of effects on measured plasma insulin concentrations in another cohort of anesthetized C57BL/6J mice when blood was sampled within short intervals from the same site (in this case from the retrobulbar sinus). 
help with the interpretation of measurement data also at potentially much later points in time.

Unwanted variability during the blood sampling process may also be introduced by comparing analyte concentrations in samples that have been collected under narcosis with analyte concentrations in samples that have been collected without prior narcosis. It is well documented that anesthetics are capable to influence intestinal motility, gastric emptying and glucose metabolism $(31,32,33)$. In our hands and using a small set of samples collected from the tail vein with or without isoflurane anesthesia in C57BL/6J mice, plasma insulin concentrations were significantly $(P<0.05)$ lower when blood was collected under isoflurane anesthesia. Nevertheless, plasma insulin concentrations measured in samples collected with the two different anesthesia protocols showed a tight and significant correlation (Fig. 2). The displayed data show an inhibitory effect of isoflurane narcosis on plasma insulin secretion, which is in accordance with the majority of published studies $(32,33,34,35,36,37,38)$. Interestingly, Zardooz et al. described in male rats that the effect of isoflurane exposure on plasma insulin concentrations seems to depend on the fasting state, as they could not detect effects of the isoflurane anesthesia on plasma insulin in the fed state

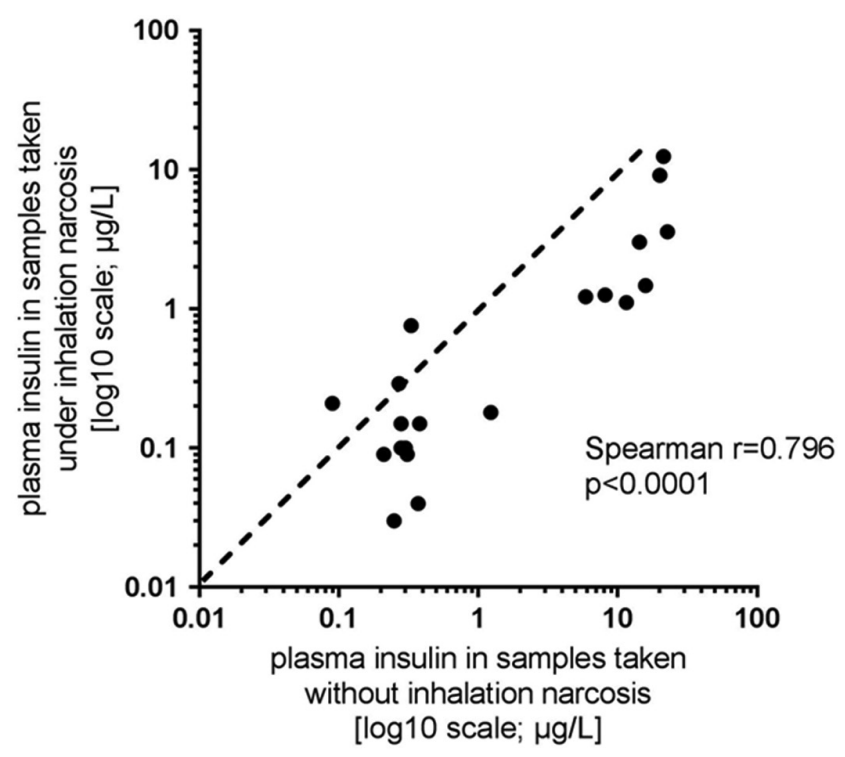

Figure 2

Comparison of measured plasma insulin concentrations in samples collected from the tail vein with or without prior isoflurane anesthesia in C57BL/6J mice. The anesthetic procedure before blood sampling was alternated from mouse to mouse to avoid the introduction of a systematic error. In this example, measured plasma insulin concentrations were significantly $(P<0.05)$ lower when blood was collected under isoflurane anesthesia but showed a tight and significant non-parametric correlation.
(39). The effect of isoflurane narcosis on plasma insulin has been found to be even more pronounced when glucosestimulated insulin concentrations are measured, e.g. as usually done during an oral glucose tolerance test. This effect is probably best explained by the inhibitory effect of isoflurane on gastric emptying and intestinal motility, thus affecting the passage time of the orally administered glucose $(33,35,36)$.

In contrast to the parameter 'rodent insulin', where already several groups reported clear effects of the narcosis protocol on the circulating concentrations, many other metabolic hormones in rodents unfortunately lack this type of systematic studies. It may be assumed that the type of narcosis and the sampling site can affect the measurement result of many other metabolic hormones. However, there are also examples from relatively stable circulating parameters in rodents, such as IGF-I, which seems to be only marginally affected by these variables ((40) and unpublished observations). Unfortunately, there is no strict rule that can predict if or in which direction a specific narcosis protocol or sampling site affects the analyte of interest. The easiest and best way to circumvent this potential source of unwanted biological variability in rodent studies is to stick exactly to the same sampling procedures, including the usage of the same equipment (e.g. blood sampling devices) needed to collect the sample, as well as to only compare samples from animals that underwent identical pre-treatment procedures. Of course, easily controllable biological variables such as the fasting condition before sampling, e.g. during dynamic tolerance tests, should be kept constant between experimental groups throughout the enite study. Further guidance and recommendations on this topic can also be found in recent comprehensive literature reports $(41,42)$.

\section{Sample processing}

After sample collection, the 'pre-analytical pitfall journey' continues. Blood samples should be collected in suitable containers to obtain plain serum or alternatively in containers pre-filled with anti-coagulants such as EDTA, sodium citrate or heparin to obtain plasma. Many sources recommend the usage of either serum or heparin- or EDTA-plasma for routine biochemistry analyses $(16,43)$, which - in principle - are also both appropriate for most of the analyses of metabolic hormones in rodents. However, there are several analytes where serum or plasma should be preferred. For example, it has been reported that bile acids concentrations are about $60 \%$ lower when measured in

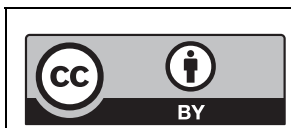

This work is licensed under a Creative Commons Attribution 4.0 International License. 
heparin-plasma compared to serum (44). Trace elements like $\mathrm{Zn}$ or $\mathrm{Mg}$ can only be analyzed in serum, because in plasma, the ions form complexes with the chelating agents such as EDTA or heparin due to their bivalent nature (45). In contrast, some peptide hormones like the pituitary-derived ACTH should be measured in plasma for stability reasons (46).

It may also be useful to add an unspecific protease inhibitor to the blood sample or to pre-load the sample collection tube with such an inhibitor. This can be recommended particularly if the complete list of analytes is not yet specified at the time of sampling or if it is already known that analytes are susceptible to degradation by proteases (e.g. active GLP-1). Furthermore, it is important to check if further 'additives' are required to stabilize the analyte. One example for an analyte requiring a specific sampling protocol is acylated ghrelin, because here, acidification of the sample through addition of $\mathrm{HCl}$ is required to preserve the octanoyl group of ghrelin $(47,48)$. Depending on the number and stability of the analytes of interest, it might be required to collect several aliquots with different additives to obtain the optimal sample type for each analyte. Unfortunately, such an approach is often not possible in rodent studies because of the limitations in sampling volume. However, since the type of matrix as well as the sample pre-treatment conditions can have a significant impact on the immunoassay measurement result, a minimum requirement is that only data from samples which were collected and processed with the same pre-treatment protocols should be compared.

In previous experiments in rats (9), we have demonstrated that the centrifugation protocol had only limited or no effect on the measurement results of five different metabolic hormones, including insulin and GLP-1. However, the lack of effects of the centrifugation protocol on these randomly selected hormones should not to be taken as a generalized rule for all metabolic hormones in each rodent species. Again, most important is consistency in all pre-analytical procedures throughout one set of experiments. After centrifugation, serum or plasma samples must be stored in appropriate tubes (not to forget a clear and wash-off resistant sample identifier) with appropriate lids (proper sealing of the tubes can prevent evaporation/contamination of the sample). If immunoassay measurements cannot be done right away, (long-term) storage should be carried out in the freezer, ideally at $-70^{\circ} \mathrm{C}$ or lower, again to prevent proteolytic activity and sample evaporation. Frequent freeze-thaw cycles can also seriously affect analyte concentrations (9) and thus should be avoided. If multiple analytes are to be measured using singleplex assays, it is advisable to prepare multiple aliquots of the sample.

Realizing the many steps that are necessary to obtain the final matrix in which the analyte of interest will be measured, it appears obvious that these steps can vary between different research groups and labs. Luckily, the many different possibilities of sample collection, processing and storage are not a too serious issue in preclinical rodent research as long as conditions are kept standardized and consistent within an experimental series and as long as all samples undergo exactly the same procedures before measurement. It is appeasing to realize that any potential measurement variability introduced by one or multiple specific pre-analytical procedure(s) can be considered to be of minor relevance in this context because this 'bias' should - in theory - affect the measurement results of all samples in the same direction and in a similar order of magnitude. Overall, the relative differences (or lack of differences) between control and observer groups within one experiment should remain constant if the above described general rules are applied. Nevertheless, it is very important to keep in mind that the pre-analytical phase has a significant impact on immunoassay measurements of rodent samples, also in order to avoid an unintended mixture of pre-analytical procedures between samples from the same experimental run. Of note, it is also likely that the selection of specific procedures during the pre-analytical phase contributes to differences in reported concentrations for the same hormones analyzed by different groups or by different labs.

\section{Analytical variability}

Knowledge about the existence of analytical variability and a basic understanding of how to control this variable is of great importance to conduct meaningful interpretations of measured values from rodent samples. However, a comprehensive discussion of the complex topics 'analytical variability' and 'measurement uncertainty' in immunoassay measurements is beyond the scope of this review, and readers may be referred to more detailed literature for further insights $(49,50,51,52)$. Instead, the following section will summarize some considerations, which are of importance in the context of immunoassay measurement of metabolic hormones in rodents. http://www.endocrineconnections.org
https://doi.org/10.1530/EC-18-0035
(C) 2018 The authors Published by Bioscientifica Ltd

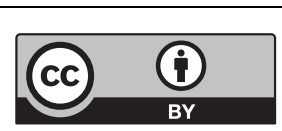

This work is licensed under a Creative Commons Attribution 4.0 International License. 


\section{Intra- and inter-assay variability in rodent immunoassays}

Although the quality criteria for immunoassays used 'for research purposes only' (RUO) obviously can be less strict compared to immunoassays used for clinical diagnostics in human, also immunoassays used for the measurement of metabolic hormones in rodents should - in principle - follow the same guidelines and recommendations. Among others, the Clinical and Laboratory Standards Institute published recommendations with respect to acceptable limits of detection and quantification, imprecision, linearity and recovery $(53,54)$. The chosen analytical method (e.g. mass spectrometry or immunoassay) can significantly affect the measurement result, but also different types of the same method, e.g. sandwich-type or competitive immunoassays or assays from different manufacturers for the same analyte, likely will result in differences of reported absolute concentrations. Lack of standardization or harmonization has been recognized as one of the most relevant issues in diagnostic procedures used in clinical medicine. However, the lack of standardization is even more common in the less regulated market of RUO reagents. For example, we recently have shown considerable differences in assay readouts when IGF-I was measured in serum of mice with two different mouse-specific IGF-I immunoassays (9). Similar examples of a significant variability for the same analyte between rodent assays from different vendors are documented throughout literature. For the purpose of this review, and using the analysis of FGF-15 in mice as one example, here we illustrate the significant performance differences between immunoassays of different vendors (Table 1). To overcome such discrepancies for assays used in clinical diagnostics in humans, standardizing and harmonizing the preparations of the reference materials, using a single, recombinant calibrator for all assays of a specific analyte and reporting in the same mass units for each analyte have been recommended $(55,56)$.
The shortcomings, but sometimes also the success of such efforts to standardize or harmonize assays in clinical labs is reflected by the results from external quality assessment schemes (EQAS), in which to participate is mandatory for clinical laboratories in most countries. This type of external quality control, however, is rarely performed for rodent research immunoassays. Luckily, the quantitative differences between assays from different manufacturers are of limited importance in most basic rodent studies as long as all samples from an experimental series have been measured in the same batch of immunoassays from the same provider - ideally in the same analytical run. In contrast to human diagnostics, where the measured concentration of an analyte is often interpreted in relation to absolute cut-off values or to analyte-specific age- and sex-matched reference intervals (and thus directly contributes to the clinical interpretation of a patient's disease state), researchers investigating metabolic hormones in rodents are usually more interested to obtain the 'relative changes' of the analyte. 'Relative' in this context means that differences in measured concentrations are compared between an observer group and the corresponding control group. However, as soon as the analytical method to measure rodent hormones in a laboratory is being changed or data from one lab are being compared to those obtained in another lab using a different kit, the methodological differences leading to differences in absolute concentrations reported need to be taken into account.

In addition to the above-mentioned 'between-assay variability' - which is attributable to differences in the assay components provided by the manufacturer and completely independent from the operator using the assay - systematic and random errors in the actual lab work can also contribute to 'between-assay variability', but also to 'within-assay' variability. Such variability is an inherent feature of any analytical procedure and relates to random variability or systematic mistakes in an operators performance of the assay, but also to variability

Table 1 Displays some basic performance characteristics of an exemplary assay comparison between immunoassays from two commercial vendors, both measuring FGF-15 in mouse plasma.

Precision (intra-assay CV) (\%)

\begin{tabular}{|c|c|}
\hline Vendor A & Vendor B \\
\hline 14.7 & 4.1 \\
\hline 380 & 60 \\
\hline 260 & 58 \\
\hline n.d. & 54 \\
\hline
\end{tabular}

Recovery at a concentration range in the upper third of the calibration curve (\%)

Intra-assay variability was determined by 6-fold analysis, recovery was analyzed as 'expected over observed' concentrations using recombinant mouse FGF-15 from an external provider. Analytes were added at the low, medium and upper end of the calibration curve. n.d.: not determined.

http://www.endocrineconnections.org

https://doi.org/10.1530/EC-18-0035
(๔) 2018 The authors Published by Bioscientifica Ltd

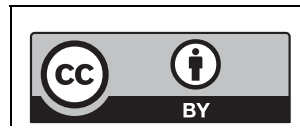

This work is licensed under a Creative Commons Attribution 4.0 International License. 
in environmental conditions like temperature, humidity as well as in the technical performance of instruments like pipettes, plate shakers or washers. This inherent variability is often summarized as 'coefficient of variability' (\% CV) or 'measurement uncertainty'. Within one immunoassay run, such variability can be controlled by measuring all samples in duplicate or triplicate, a measure which although expensive - should also be applied to rodent samples. Although the maximum acceptable degree of variability depends on the specific analyte and the specific research question, it is a common recommendation that the $\mathrm{CV} \%$ for a duplicate analysis should not exceed $15 \%$ otherwise the measurement should be repeated. In case the available sample volume is too low, and provided that the immunoassay for the desired analyte is sensitive enough, one might consider diluting the sample with a suitable diluent in order to achieve duplicate measurements or to repeat the measurement. The impact of environmental conditions is much harder to predict and to control. However, it is recommended to monitor and document some of the basic conditions during an assay run to allow retrospective identification of factors negatively affecting assay performance. In the clinical laboratory, the concept of internal quality control samples analyzed with each assay has been implemented to control between-assay variability. Manufacturers of rodent assays sometimes provide such control samples. However, it should be carefully examined by each user of the assay if these quality control samples are suitable to monitor assay performance in a concentration range, which is meaningful to the outcome of a rodent study. Most assays perform comparably well in the mid-range of the calibration curve, while the performance is less reliable toward the lower or upper end of the standard curve concentration ranges, which might be very relevant for a scientific conclusion from an experiment. It should also be checked whether the quality control material provided with an assay mirrors 'real samples' in terms of matrix. It is common that so-called 'control samples' just are made of one of the calibrators. It depends on the matrix of the real samples whether such an approach is sufficient to monitor assay quality. To control assay performance over a larger series of immunoassay plates it might be advisable to have your own 'control sample' running with each assay. Such samples can be produced from leftovers samples from previous experiments and should be separated into a sufficient number of aliquots to allow measuring one of the 'real samples' with each assay. This allows calculation of the CV\% over the measurement

http://www.endocrineconnections.org https://doi.org/10.1530/EC-18-0035

C) 2018 The authors Published by Bioscientifica Ltd series, thereby giving an estimate of the uncertainty associated with the generation of the data.

\section{Basic assay validation steps for rodent immunoassays}

It is fascinating to realize that nature has implemented such a complex set of regulatory factors controlling metabolism and new, previously unknown circulating metabolic hormones are discovered in periodic intervals. In addition, known hormones can also become a 'hot-topic' with the need to develop novel or more sensitive immunoassays if research reveals a previously unrecognized (maybe broader) impact of this specific hormone in other relevant scientific areas such as metabolic research. Examples for recently discovered or recognized metabolic hormones include Asprosin, the glycoprotein CHI3L1 (chitinase-3-like protein 1), GDF15, Phoenixin or members of the fibroblast growth factor family $(57,58,59,60,61,62,63,64,65,66,67,68,69)$. In order to also offer the required research tools, providers sell analyte-specific immunoassays for rodents usually soon after the sequence/structure of the new hormone has become available or if a relevant sales market is expected. It is sometimes surprising to see how quickly after the discovery process particularly smaller biotech companies manage to offer immunoassays for the new analytes.

As mentioned earlier, FGF-15 is an example of a metabolic hormone that has emerged as a new, potentially interesting factor controlling body weight and glucose metabolism. Here, assay performance data of two mousespecific FGF-15 immunoassays from two different vendors are displayed for the parameters 'precision', 'recovery' and 'linearity'. Of note, the assay manufacturers/ vendors of these two mouse FGF-15 immunoassays are intentionally not named as this example is meant to increase the awareness for analytical differences between immunoassays from different sources rather than to provide recommendations for a specific mouse FGF-15 immunoassay. Table 1 illustrates for this exemplary assay comparison that the robustness of measurement results as well as the overall assay performance clearly depends on the chosen immunoassay and obviously, both assays could be further optimized. The idea of conducting this type of basic assay validation is to provide guidance in case a researcher needs to opt between two (or more) available immunoassay options for a specific analyte. Furthermore, such a basic assay validation will also yield 
important data to decide if the chosen, newly established immunoassay is capable to deliver robust and meaningful data. Important steps of this basic validation include the analysis of precision and linearity; the latter can for example be done with serial dilutions of the calibrators or positive controls, which should always be delivered with an immunoassay kit. Other recent examples of hormonespecific and extended, state-of-the-art assay validation efforts for novel immunoassays measuring hormones in samples from laboratory animal species can be found in the cited references $(70,71,72,73,74)$. In addition, literature research or personal exchange during scientific meetings can be helpful to elucidate if other research groups have successfully used the (potentially novel) immunoassay to measure the analyte. In conclusion, before using a rodent immunoassay for the first time, it is highly recommended to invest time to check if others have effectively used a specific immunoassay for measurement of rodent samples and to include basic validation measurements, especially if the samples of interest are precious with only little available sample volumes. Ultimately, researchers understandably want to base their conclusions on meaningful measurement results aiming to avoid the generation of random numbers from precious rodent samples. In case the validation of a novel immunoassay is not successful or if data are not convincing, one may consider using alternative approaches for the measurement of the newly discovery hormone (e.g. LC-MS/MS).

\section{Multiplexed immunoassays}

A remarkable trend in preclinical research involving measurement of rodent metabolic hormones is the use of so-called multiplex(-ed) assays. These assays are based on a fascinating technology offering the potential to measure multiple, up to 100 analytes (75) in a single sample, which typically needs less than $100 \mu \mathrm{L}$ of sample volume. This technique has many advantages including the increased efficiency at a reduced expense, greater output per sample/volume ratio and a higher sample throughput $(76,77)$.

On the contrary, this technical approach also warrants some caution and comes with challenges. Cross-reactivity and the probability of cross-talking interferences of assay components, which is increasing exponentially with the number of analytes in a multiplex immunoassay (78), have been difficult to mitigate, limiting the assay performance, and thus potentially returning inaccurate or even false results (79). Also in human diagnostics, technical and operational challenges are limiting the routine implementation of multiplexed assays in clinical settings (80). The challenges of this technology may be exemplified with the fact that all analytes are being measured in the same matrix. However, the manufacturers of multiplex technology assays, especially for rodent panels, rarely provide data on how different matrix types affect the measurement of single analytes in their multiplex panels and assume that 'one matrix fits all'. As mentioned earlier, ACTH is an example of a hormone for which immunoassay measurements in plasma are recommended. However, rodent-specific multiplexed panels for hormones, including ACTH, are offered, which are described to also reliably function in serum (81). Similar to singleplex immunoassays, users of rodent multiplex immunoassays should carefully evaluate if the sample matrix has an effect on the analyte measurement. Overall, the same principles used for validation of singleplexed immunoassays also apply to this technology and a basic validation should be carried out rigorously for each analyte in a new multiplex panel (80), e.g. as conducted by Sun et al. for the simultaneous measurement of aldosterone and testosterone in mice (73). In case that the multiplex panel is pre-designed, one may use the own validation data to decide if all analytes or only a subset of analytes can be regarded to deliver reliable data.

With respect to analytical variability Figure 3 depicts (using murine plasma insulin measurements as an example) how different the measured concentrations between multiplex and singleplex immunoassays can be. Both, Passing-Bablok regression and Bland-Altman plot show, not surprisingly, that the overall correlation is relatively weak with a comparably high fit difference. Importantly, from this analysis, one cannot determine which measurement is 'right' or provides the 'true' value in plasma, because immunoassay measurements always need to be interpreted relative to a calibration curve and do not represent an absolute quantification. However, from this example, three important conclusions can be drawn with respect to using multiplex assays: (i) measurement results can differ between multiplex and singleplex assays and neither data from multiplex nor singleplex measurements should be regarded as the 'true' concentrations; (ii) data should always be compared and interpreted relative to biological controls (e.g. 'no treatment' or 'wild-type' controls) measured by the same assay method and ideally also in the same experimental run; (iii) during a series of experiments, it is mandatory to stick to the same measurement method and assay throughout.

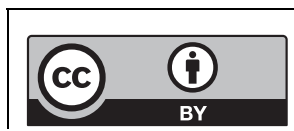

This work is licensed under a Creative Commons Attribution 4.0 International License. 

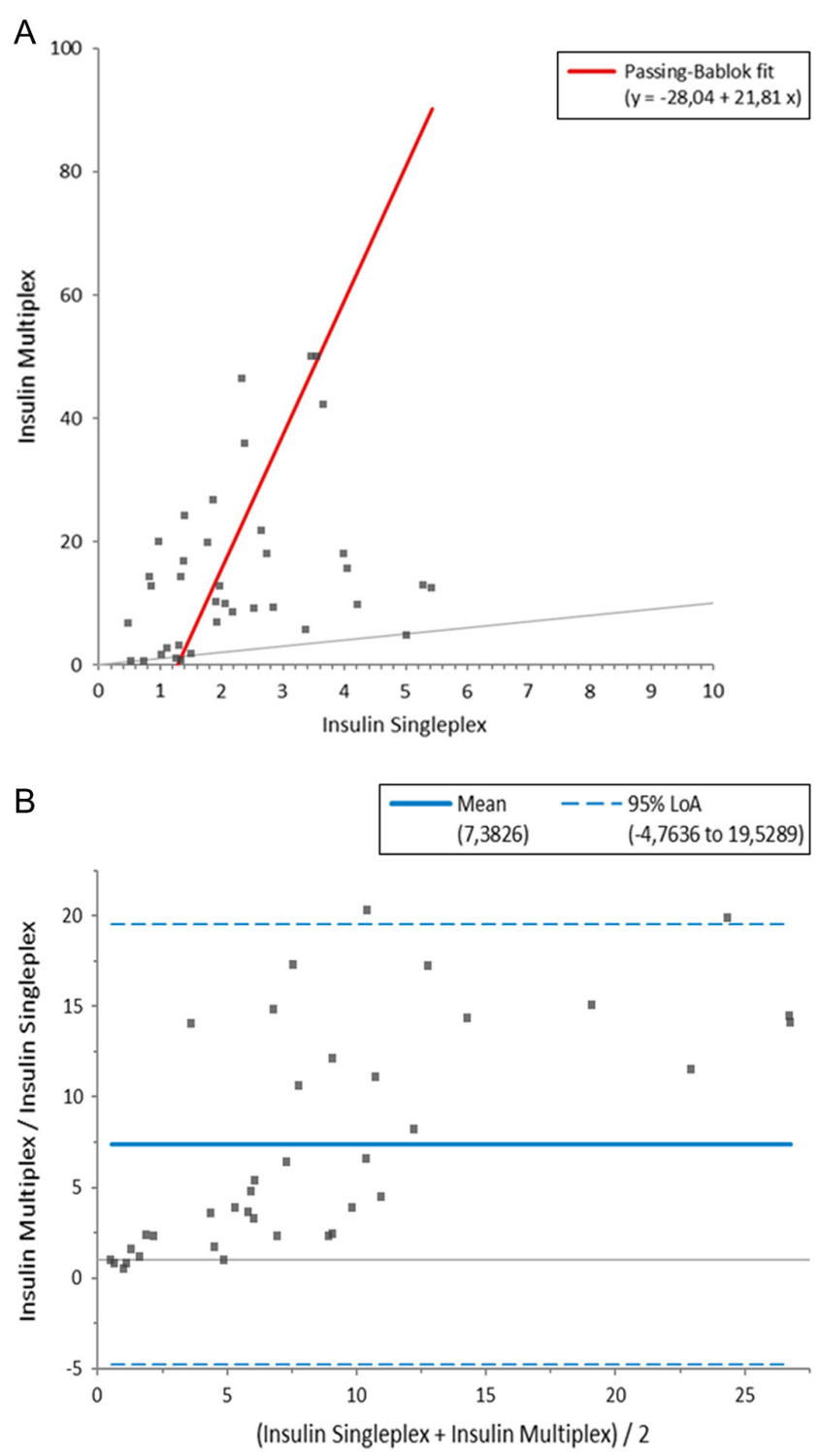

Figure 3

Plasma insulin was measured in the same 38 mouse samples by two different immunoassays (multiplex and singleplex). Measured concentrations showed a significant variability depending on the immunoassay. (A) Depicts the results using a Passing-Bablok regression and $(B)$ shows the corresponding Bland-Altman plot for this assay comparison.

\section{Concluding recommendations}

In summary, it is obvious that pre-analytical and analytical variability can significantly affect the reliability and reproducibility of data on metabolic hormones in rodents. In view of the innumerable spectrum of commercially available assays for the measurement of hormones in rodents, it is very important to bear in mind that the market for the 'research-use-only' reagents is far less strictly regulated and standardized than the market
(C) 2018 The authors Published by Bioscientifica Ltd for assays used in clinical diagnostics in humans. Most of the validation of these analytical methods remains to be done by the customer, i.e., the research lab using the assays. Having this awareness at the back of one's mind is the first crucial step for appropriate planning, and also for interpretation of metabolic studies in rodent. Notably, measures exist to control variability and to avoid frequent pitfalls, and such measures need to be implemented in an attempt to obtain high-quality data. With respect to pre-analytical variability, it is of utmost importance that all samples undergo the same standardized workflows including a careful documentation of the relevant preanalytical steps. With respect to analytical variability, concerns have been expressed that current research immunoassays could have been pushed beyond their limits. Therefore, it is a challenge for both, assay providers and customers, to rapidly find a 'balanced, equitable and scientifically sound solution' (7) to improve the performance of immunoassay measurements in endocrine research involving rodent species. In the lab, one should clearly consider conducting a basic validation of an immunoassay with leftover samples before using it for the first time with precious samples. As for the pre-analytical period, also during the analytical process, all steps should follow a standardized procedure to minimize analytical variability. In case the basic immunoassay validation experiments do not provide the expected confidence in the method, contacting manufacturers or experienced colleagues may be helpful to discriminate between problems associated with the analytical method itself on the one hand and random errors in the performance of the assay on the other. Adherence to these recommendations will help to obtain reliable and meaningful data from measurements in rodent samples, ultimately reducing unpleasant surprises, resources and frustration.

\section{Materials and methods}

\section{Analytical procedures related to the measurement of metabolic parameters in mice}

Blood samples were collected from adult male and female C57BL/6J mice (Charles River). Mice were group-housed at room temperature in an environmentally controlled SPF-animal facility with a 12-h light-darkness cycle and had access to a standard maintenance rodent diet (Ssniff, Soest, Germany) and filtered tap water ad libitum. All animal experimental procedures were approved by the internal animal welfare committee as well as by German http://www.endocrineconnections.org https://doi.org/10.1530/EC-18-0035
This work is licensed under a Creative Commons Attribution 4.0 International License. 
government authorities. Table 1 displays basic performance characteristics of an exemplary assay comparison between immunoassays from two commercial vendors, both commercialized for the measurement of FGF-15 in mouse serum. Intra-assay variability was determined by 6-fold analysis of mouse serum samples, and linearity was analyzed by measuring FGF-15 in serial dilutions of pooled mouse serum samples (using the diluent provided by the assay vendors). Recovery was analyzed as 'expected over observed' concentrations using recombinant mouse FGF-15 (\% recovery=observed concentration/expected concentration $\times 100 \%$ ). Analytes were added at the low, medium and upper end of the calibration curve. For the experiments related to different blood sampling sites, plasma insulin concentrations were measured in samples collected either via puncture of the tail vein or from the retrobulbar sinus of mice. During the whole sampling process, mice remained under isoflurane narcosis and the two blood samples were collected within 3 min per mouse. The 'first/starting' sampling site was alternated after each mouse to avoid the introduction of a timely bias. In another cohort of 20 mice, the effect of isoflurane narcosis on plasma insulin was studied. Therefore, two blood samples were collected from the tail vein of the same mouse, one time under isoflurane narcosis in an inhalation chamber or for the subsequent sampling without anesthesia in conscious mice (again alternating the timing of the procedure from mouse to mouse). All blood samples were collected with plastic capillaries coated with sodium heparin (SC-Sanguis Counting $\mathrm{GmbH}$, Nümbrecht, Germany) into prefilled EDTA tubes on ice. After the collection process, samples were routinely and uniformly processed and stored in Nunc cryotubes (Thermo Fisher Scientific GmbH) at $-80^{\circ} \mathrm{C}$ until analysis. All plasma samples underwent only a single freeze-thaw cycle and were analyzed in the same run of an immunoassay specific for the measurement of rodent insulin (mouse/rat insulin, Meso Scale Discovery, Gaithersburg, MD, USA). Results are depicted in Figs 1 and 2.

For the comparison of insulin single vs multiplexed immunoassays (Fig. 3), 38 plasma samples from mice were measured in duplicate and in parallel in two different mouse insulin assays (multiplex assay: Merck Millipore, Milliplex MAP Kit, Mouse Metabolic Magnetic Bead Panel, MMHMAG-44K; singleplex assay: Meso Scale Discovery, Mouse Insulin Assay, K152BZC-2). The comparison between the two immunoassay measurement methods (Passing-Bablok regression and Bland-Altman plot) has been carried out using the software Analyse-It (Analyse-it Software, Ltd., Leeds, United Kingdom, Version 4.80.2).

\section{Declaration of interest}

Max Bielohuby and Uwe Schwahn are employees of Sanofi-Aventis Deutschland $\mathrm{GmbH}$.

\section{Funding}

This work did not receive any specific grant from any funding agency in the public, commercial, or not-for-profit sector.

\section{References}

1 Yalow RS \& Berson SA. Assay of plasma insulin in human subjects by immunological methods. Nature 1959184 (Supplement 21) 1648-1649. (https://doi.org/10.1038/1841648b0)

2 Morgan CR \& Lazarow A. Immunoassay of insulin: two antibody system: plasma insulin levels of normal, subdiabetic and diabetic rats. Diabetes 196312 115-126. (https://doi.org/10.2337/ diab.12.2.115)

3 Muller EE, Miedico D, Giustina G, Pecile A, Cocchi D \& Mandelli V. Impaired secretion of growth hormone in goldthioglucose-obese mice. Endocrinology 197189 56-62. (https://doi.org/10.1210/endo-891-56)

4 Schindler WJ, Hutchins MO \& Septimus EJ. Growth hormone secretion and control in the mouse. Endocrinology 197291 483-490. (https://doi.org/10.1210/endo-91-2-483)

5 Engvall E \& Perlmann P. Enzyme-linked immunosorbent assay (ELISA). Quantitative assay of immunoglobulin G. Immunochemistry 19718 871-874. (https://doi.org/10.1016/0019-2791(71)90454-X)

6 Van Weemen BK \& Schuurs AH. Immunoassay using antigenenzyme conjugates. FEBS Letters 197115 232-236. (https://doi. org/10.1016/0014-5793(71)80319-8)

7 Auchus RJ. Steroid assays and endocrinology: best practices for basic scientists. Endocrinology 2014155 2049-2051. (https://doi. org/10.1210/en.2014-7534)

8 Christenson RH \& Duh SH. Methodological and analytic considerations for blood biomarkers. Progress in Cardiovascular Diseases 201255 25-33. (https://doi.org/10.1016/j.pcad.2012.05.001)

9 Bielohuby M, Popp S \& Bidlingmaier M. A guide for measurement of circulating metabolic hormones in rodents: pitfalls during the pre-analytical phase. Molecular Metabolism 20121 47-60. (https://doi. org/10.1016/j.molmet.2012.07.004)

10 Agrawal L, Engel KB, Greytak SR \& Moore HM. Understanding preanalytical variables and their effects on clinical biomarkers of oncology and immunotherapy. Seminars in Cancer Biology 2017 [epub]. (https://doi.org/10.1016/j.semcancer.2017.12.008)

11 Lippi G, Banfi G, Buttarello M, Ceriotti F, Daves M, Dolci A, Caputo M, Giavarina D, Montagnana M, Miconi V, et al. Recommendations for detection and management of unsuitable samples in clinical laboratories. Clinical Chemistry and Laboratory Medicine 200745 728-736. (https://doi.org/10.1515/CCLM.2007.195)

12 Lippi G, Guidi GC, Mattiuzzi C \& Plebani M. Preanalytical variability: the dark side of the moon in laboratory testing. Clinical Chemistry and Laboratory Medicine 200644 358-365. (https://doi. org/10.1515/CCLM.2006.073)

13 Young DS. Conveying the importance of the preanalytical phase. Clinical Chemistry and Laboratory Medicine 200341 884-887. (https:// doi.org/10.1515/CCLM.2003.133)

14 Laron Z, Bidlingmaier M \& Strasburger CJ. Indications, limitations and pitfalls in the determination of human growth hormone, IGF-I and their binding proteins. Pediatric Endocrinology Reviews 20075 (Supplement 1) 555-569.

15 Schilbach K, Strasburger CJ \& Bidlingmaier M. Biochemical investigations in diagnosis and follow up of acromegaly. Pituitary 201720 33-45. (https://doi.org/10.1007/s11102-017-0792-z)

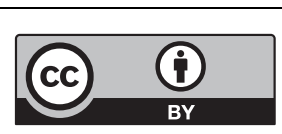

This work is licensed under a Creative Commons Attribution 4.0 International License. 
16 Braun JP, Bourges-Abella N, Geffre A, Concordet D \& Trumel C. The preanalytic phase in veterinary clinical pathology. Veterinary Clinical Pathology 201544 8-25. (https://doi.org/10.1111/vcp.12206)

17 Hernandes VV, Barbas C \& Dudzik D. A review of blood sample handling and pre-processing for metabolomics studies. Electrophoresis 201738 2232-2241. (https://doi.org/10.1002/elps.201700086)

18 Janssen K \& Delanghe J. Importance of the pre-analytical phase in blood glucose analysis. Acta Clinica Belgica 201065 311-318. (https://doi.org/10.1179/acb.2010.068)

19 Jensen AL, Wenck A, Koch J \& Poulsen JS. Comparison of results of haematological and clinical chemical analyses of blood samples obtained from the cephalic and external jugular veins in dogs. Research in Veterinary Science 199456 24-29. (https://doi. org/10.1016/0034-5288(94)90191-0)

20 Lima-Oliveira G, Volanski W, Lippi G, Picheth G \& Guidi GC. Pre-analytical phase management: a review of the procedures from patient preparation to laboratory analysis. Scandinavian Journal of Clinical and Laboratory Investigation 201777 153-163. (https://doi.org /10.1080/00365513.2017.1295317)

21 Aasland KE, Skjerve E \& Smith AJ. Quality of blood samples from the saphenous vein compared with the tail vein during multiple blood sampling of mice. Laboratory Animals 201044 25-29. (https://doi. org/10.1258/la.2009.009017)

22 Christensen SD, Mikkelsen LF, Fels JJ, Bodvarsdottir TB \& Hansen AK. Quality of plasma sampled by different methods for multiple blood sampling in mice. Laboratory Animals 200943 65-71. (https://doi. $\operatorname{org} / 10.1258 /$ la.2008.007075)

23 Hoggatt J, Hoggatt AF, Tate TA, Fortman J \& Pelus LM. Bleeding the laboratory mouse: not all methods are equal. Experimental Hematology 201644 132.e131-137.e131. (https://doi.org/10.1016/j. exphem.2015.10.008)

24 Schnell MA, Hardy C, Hawley M, Propert KJ \& Wilson JM. Effect of blood collection technique in mice on clinical pathology parameters. Human Gene Therapy 200213 155-161. (https://doi. org/10.1089/10430340152712700)

25 Fernandez I, Pena A, Del Teso N, Perez V \& Rodriguez-Cuesta J. Clinical biochemistry parameters in C57BL/6J mice after blood collection from the submandibular vein and retroorbital plexus. Journal of the American Association for Laboratory Animal Science 2010 49 202-206.

26 Grouzmann E, Cavadas C, Grand D, Moratel M, Aubert JF, Brunner HR \& Mazzolai L. Blood sampling methodology is crucial for precise measurement of plasma catecholamines concentrations in mice. Pflügers Archiv 2003447 254-258. (https://doi.org/10.1007/ s00424-003-1140-x)

27 Lee G \& Goosens KA. Sampling blood from the lateral tail vein of the rat. Journal of Visualized Experiments 201599 e52766. (https://doi. org/10.3791/52766)

28 Spencer RL \& Deak T. A users guide to HPA axis research. Physiology and Behavior 2017178 43-65. (https://doi.org/10.1016/j. physbeh.2016.11.014)

29 Teilmann AC, Kalliokoski O, Sorensen DB, Hau J \& Abelson KS. Manual versus automated blood sampling: impact of repeated blood sampling on stress parameters and behavior in male NMRI mice. Laboratory Animals 201448 278-291. (https://doi. org/10.1177/0023677214541438)

30 Vahl TP, Ulrich-Lai YM, Ostrander MM, Dolgas CM, Elfers EE, Seeley RJ, D'Alessio DA \& Herman JP. Comparative analysis of ACTH and corticosterone sampling methods in rats. American Journal of Physiology: Endocrinology and Metabolism 2005289 E823-E828. (https://doi.org/10.1152/ajpendo.00122.2005)

31 Goldfine ID \& Arieff AI. Rapid inibition of basal and glucosestimulated insulin release by xylazine. Endocrinology $1979 \mathbf{1 0 5}$ 920-922. (https://doi.org/10.1210/endo-105-4-920)

32 Pomplun D, Mohlig M, Spranger J, Pfeiffer AF \& Ristow M. Elevation of blood glucose following anaesthetic treatment in C57BL/6 mice. Hormone and Metabolic Research 200436 67-69. (https://doi. org/10.1055/s-2004-814104)

33 Windelov JA, Pedersen J \& Holst JJ. Use of anesthesia dramatically alters the oral glucose tolerance and insulin secretion in $\mathrm{C} 57 \mathrm{Bl} / 6$ mice. Physiological Reports 20164 e12824. (https://doi.org/10.14814/ phy2.12824)

34 Sano Y, Ito S, Yoneda M, Nagasawa K, Matsuura N, Yamada Y, Uchinaka A, Bando YK, Murohara T \& Nagata K. Effects of various types of anesthesia on hemodynamics, cardiac function, and glucose and lipid metabolism in rats. American Journal of Physiology: Heart and Circulatory Physiology 2016311 H1360-H1366. (https://doi. org/10.1152/ajpheart.00181.2016)

35 Tanaka K, Kawano T, Tomino T, Kawano H, Okada T, Oshita S, Takahashi A \& Nakaya Y. Mechanisms of impaired glucose tolerance and insulin secretion during isoflurane anesthesia. Anesthesiology 2009111 1044-1051. (https://doi.org/10.1097/ ALN.0b013e3181bbcb0d)

36 Tanaka K, Kawano T, Tsutsumi YM, Kinoshita M, Kakuta N, Hirose K, Kimura M \& Oshita S. Differential effects of propofol and isoflurane on glucose utilization and insulin secretion. Life Sciences 201188 96-103. (https://doi.org/10.1016/j.lfs.2010.10.032)

37 Wren-Dail MA, Dauchy RT, Blask DE, Hill SM, Ooms TG, Dupepe LM \& Bohm RP Jr. Effect of isoflurane anesthesia on circadian metabolism and physiology in rats. Comparative Medicine 201767 138-146.

38 Zuurbier CJ, Keijzers PJ, Koeman A, Van Wezel HB \& Hollmann MW. Anesthesia's effects on plasma glucose and insulin and cardiac hexokinase at similar hemodynamics and without major surgical stress in fed rats. Anesthesia and Analgesia 2008106 135-142. (https://doi.org/10.1213/01.ane.0000297299.91527.74)

39 Zardooz H, Rostamkhani F, Zaringhalam J \& Faraji Shahrivar F. Plasma corticosterone, insulin and glucose changes induced by brief exposure to isoflurane, diethyl ether and $\mathrm{CO}_{2}$ in male rats. Physiological Research 201059 973-978.

40 Bielohuby M, Popp S \& Bidlingmaier M. Influence of pre-analytical conditions on the measurement of components of the GH/IGF axis in rats. Growth Hormone and IGF Research 201323 141-148. (https:// doi.org/10.1016/j.ghir.2013.05.001)

41 Ayala JE, Samuel VT, Morton GJ, Obici S, Croniger CM, Shulman GI, Wasserman DH, McGuinness OP \& Consortium NIHMMPC. Standard operating procedures for describing and performing metabolic tests of glucose homeostasis in mice. Disease Models and Mechanisms 2010 3 525-534. (https://doi.org/10.1242/dmm.006239)

42 Bowe JE, Franklin ZJ, Hauge-Evans AC, King AJ, Persaud SJ \& Jones PM. Metabolic phenotyping guidelines: assessing glucose homeostasis in rodent models. Journal of Endocrinology 2014222 G13-G25. (https://doi.org/10.1530/JOE-14-0182)

43 Vap LM, Harr KE, Arnold JE, Freeman KP, Getzy K, Lester S, Friedrichs KR \& American Society for Veterinary Clinical P. ASVCP quality assurance guidelines: control of preanalytical and analytical factors for hematology for mammalian and nonmammalian species, hemostasis, and crossmatching in veterinary laboratories. Veterinary Clinical Pathology 201241 8-17. (https://doi.org/10.1111/j.1939165X.2012.00413.x)

44 Miles RR, Roberts RF, Putnam AR \& Roberts WL. Comparison of serum and heparinized plasma samples for measurement of chemistry analytes. Clinical Chemistry 200450 1704-1706. (https:// doi.org/10.1373/clinchem.2004.036533)

45 Ritter C, Ghahramani M \& Marsoner HJ. More on the measurement of ionized magnesium in whole blood. Scandinavian Journal of Clinical and Laboratory Investigation Supplement 1996224 275-280. (https://doi.org/10.3109/00365519609088647)

46 Reisch N, Reincke M \& Bidlingmaier M. Preanalytical stability of adrenocorticotropic hormone depends on time to centrifugation rather than temperature. Clinical Chemistry 200753 358-359. (https://doi.org/10.1373/clinchem.2006.080622)

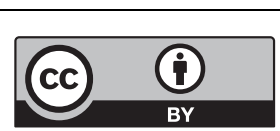

This work is licensed under a Creative Commons Attribution 4.0 International License. 
47 Prudom C, Liu J, Patrie J, Gaylinn BD, Foster-Schubert KE, Cummings DE, Thorner MO \& Geysen HM. Comparison of competitive radioimmunoassays and two-site sandwich assays for the measurement and interpretation of plasma ghrelin levels. Journal of Clinical Endocrinology and Metabolism 201095 2351-2358. (https:// doi.org/10.1210/jc.2009-2407)

48 Stengel A, Keire D, Goebel M, Evilevitch L, Wiggins B, Tache Y \& Reeve JR Jr. The RAPID method for blood processing yields new insight in plasma concentrations and molecular forms of circulating gut peptides. Endocrinology 2009150 5113-5118. (https://doi. org/10.1210/en.2009-0697)

49 Petersen PH, Ricos C, Stockl D, Libeer JC, Baadenhuijsen H, Fraser C $\&$ Thienpont L. Proposed guidelines for the internal quality control of analytical results in the medical laboratory. European Journal of Clinical Chemistry and Clinical Biochemistry 199634 983-999.

50 Plebani M. Errors in clinical laboratories or errors in laboratory medicine? Clinical Chemistry and Laboratory Medicine 200644 750-759. (https://doi.org/10.1515/CCLM.2006.123)

51 Strasburger CJ \& Bidlingmaier M. How robust are laboratory measures of growth hormone status? Hormone Research $20056 \mathbf{6 4}$ (Supplement 2) 1-5. (https://doi.org/10.1159/000087745)

52 Sturgeon CM \& Viljoen A. Analytical error and interference in immunoassay: minimizing risk. Annals of Clinical Biochemistry 2011 48 418-432. (https://doi.org/10.1258/acb.2011.011073)

53 NCCLS. Evaluation of Precision Performance of Quantitative Measurement Methods; Approved Guideline, 2nd ed. Ed NCCLS. Wayne, PA, USA: NCCLS2004.

54 NCCLS. Protocols for Determination of Limits of Detection and Limits of Quantitation; Approved Guideline. Ed NCCLS. Wayne, PA, USA: NCCLS2004.

55 Bidlingmaier M. Problems with GH assays and strategies toward standardization. European Journal of Endocrinology 2008159 (Supplement 1) S41-S44. (https://doi.org/10.1530/EJE-08-0284)

56 Bidlingmaier M \& Strasburger CJ. Growth hormone assays: current methodologies and their limitations. Pituitary 200710 115-119. (https://doi.org/10.1007/s11102-007-0030-1)

57 Bhatnagar S, Damron HA \& Hillgartner FB. Fibroblast growth factor-19, a novel factor that inhibits hepatic fatty acid synthesis. Journal of Biological Chemistry 2009284 10023-10033. (https://doi. org/10.1074/jbc.M808818200)

58 Bielohuby M, Menhofer D, Kirchner H, Stoehr BJ, Muller TD, Stock P, Hempel M, Stemmer K, Pfluger PT, Kienzle E, et al. Induction of ketosis in rats fed low-carbohydrate, high-fat diets depends on the relative abundance of dietary fat and protein. American Journal of Physiology: Endocrinology and Metabolism 2011300 E65-E76. (https:// doi.org/10.1152/ajpendo.00478.2010)

59 Duerrschmid C, He Y, Wang C, Li C, Bournat JC, Romere C, Saha PK, Lee ME, Phillips KJ, Jain M, et al. Asprosin is a centrally acting orexigenic hormone. Nature Medicine 201723 1444-1453. (https:// doi.org/10.1038/nm.4432)

60 Fang S, Suh JM, Reilly SM, Yu E, Osborn O, Lackey D, Yoshihara E, Perino A, Jacinto S, Lukasheva Y, et al. Intestinal FXR agonism promotes adipose tissue browning and reduces obesity and insulin resistance. Nature Medicine 201521 159-165. (https://doi. $\operatorname{org} / 10.1038 / \mathrm{nm} .3760)$

61 Kharitonenkov A, Shiyanova TL, Koester A, Ford AM, Micanovic R, Galbreath EJ, Sandusky GE, Hammond LJ, Moyers JS, Owens RA, et al. FGF-21 as a novel metabolic regulator. Journal of Clinical Investigation 2005115 1627-1635. (https://doi.org/10.1172/ JCI23606)

62 Marcelin G, Jo YH, Li X, Schwartz GJ, Zhang Y, Dun NJ, Lyu RM, Blouet C, Chang JK \& Chua S Jr. Central action of FGF19 reduces hypothalamic AGRP/NPY neuron activity and improves glucose metabolism. Molecular Metabolism 20143 19-28. (https://doi. org/10.1016/j.molmet.2013.10.002)
63 Muller TD \& Tschop MH. Play down protein to play up metabolism? Journal of Clinical Investigation 2014124 3691-3693. (https://doi. org/10.1172/JCI77508)

64 Romere C, Duerrschmid C, Bournat J, Constable P, Jain M, Xia F, Saha PK, Del Solar M, Zhu B, York B, et al. Asprosin, a fasting-induced glucogenic protein hormone. Cell 2016165 566-579. (https://doi. org/10.1016/j.cell.2016.02.063)

65 Stejskal D, Karpisek M, Hanulova Z \& Stejskal P. Fibroblast growth factor-19: development, analytical characterization and clinical evaluation of a new ELISA test. Scandinavian Journal of Clinical and Laboratory Investigation 200868 501-507. (https://doi. org/10.1080/00365510701854967)

66 Yosten GL, Lyu RM, Hsueh AJ, Avsian-Kretchmer O, Chang JK, Tullock CW, Dun SL, Dun N \& Samson WK. A novel reproductive peptide, phoenixin. Journal of Neuroendocrinology 201325 206-215. (https://doi.org/10.1111/j.1365-2826.2012.02381.x)

67 Yuan T, Sun Z, Zhao W, Wang T, Zhang J \& Niu D. Phoenixin: a newly discovered peptide with multi-functions. Protein and Peptide Letters 201724 472-475. (https://doi.org/10.2174/092986652466617 0207154417)

68 Emmerson PJ, Wang F, Du Y, Liu Q, Pickard RT, Gonciarz MD, Coskun T, Hamang MJ, Sindelar DK, Ballman KK, et al. The metabolic effects of GDF15 are mediated by the orphan receptor GFRAL. Nature Medicine 201723 1215-1219. (https://doi.org/10.1038/nm.4393)

69 Gorgens SW, Eckardt K, Elsen M, Tennagels N \& Eckel J. Chitinase3-like protein 1 protects skeletal muscle from TNFalpha-induced inflammation and insulin resistance. Biochemical Journal 2014459 479-488. (https://doi.org/10.1042/BJ20131151)

70 Bielohuby M, Zarkesh-Esfahani SH, Manolopoulou J, Wirthgen E, Walpurgis K, Toghiany Khorasgani M, Aghili ZS, Wilkinson IR, Hoeflich A, Thevis M, et al. Validation of serum IGF-I as a biomarker to monitor the bioactivity of exogenous growth hormone agonists and antagonists in rabbits. Disease Models and Mechanisms 20147 1263-1273. (https://doi.org/10.1242/dmm.016519)

71 Handelsman DJ, Jimenez M, Singh GK, Spaliviero J, Desai R \& Walters KA. Measurement of testosterone by immunoassays and mass spectrometry in mouse serum, testicular, and ovarian extracts. Endocrinology 2015156 400-405. (https://doi.org/10.1210/en.20141664)

72 Manolopoulou J, Bielohuby M, Caton SJ, Gomez-Sanchez CE, Renner-Mueller I, Wolf E, Lichtenauer UD, Beuschlein F, Hoeflich A \& Bidlingmaier M. A highly sensitive immunofluorometric assay for the measurement of aldosterone in small sample volumes: validation in mouse serum. Journal of Endocrinology 2008196 215-224. (https:// doi.org/10.1677/JOE-07-0134)

73 Sun M, Manolopoulou J, Spyroglou A, Beuschlein F, Hantel C, Wu Z, Bielohuby M, Hoeflich A, Liu C \& Bidlingmaier M. A microsphere-based duplex competitive immunoassay for the simultaneous measurements of aldosterone and testosterone in small sample volumes: validation in human and mouse plasma. Steroids 201075 1089-1096. (https://doi.org/10.1016/j. steroids.2010.07.005)

74 van Genderen FT, Gorus FK, Pipeleers DG \& van Schravendijk CF. Sensitive and specific time-resolved fluorescence immunoassay of rat C-peptide for measuring hormone secretory and storage capacity of beta-cells in vivo and in vitro. Endocrinology 2013154 1934-1939. (https://doi.org/10.1210/en.2012-2167)

75 Houser B. Bio-Rad's Bio-Plex(R) suspension array system, xMAP technology overview. Archives of Physiology and Biochemistry 2012118 192-196. (https://doi.org/10.3109/13813455.2012.705301)

76 Leng SX, McElhaney JE, Walston JD, Xie D, Fedarko NS \& Kuchel GA. ELISA and multiplex technologies for cytokine measurement in inflammation and aging research. Journals of Gerontology: Series A, Biological Sciences and Medical Science 200863 879-884. (https://doi. org/10.1093/gerona/63.8.879) http://www.endocrineconnections.org https://doi.org/10.1530/EC-18-0035
() 2018 The authors Published by Bioscientifica Ltd

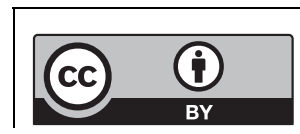

This work is licensed under a Creative Commons Attribution 4.0 International License. 
77 Tighe PJ, Ryder RR, Todd I \& Fairclough LC. ELISA in the multiplex era: potentials and pitfalls. Proteomics: Clinical Applications 20159 406-422. (https://doi.org/10.1002/prca.201400130)

78 Marquette CA, Corgier BP \& Blum LJ. Recent advances in multiplex immunoassays. Bioanalysis 20124 927-936. (https://doi.org/10.4155/ bio.12.56)

79 Juncker D, Bergeron S, Laforte V \& Li H. Cross-reactivity in antibody microarrays and multiplexed sandwich assays: shedding light on the dark side of multiplexing. Current Opinion in Chemical Biology 201418 29-37. (https://doi.org/10.1016/j. cbpa.2013.11.012)

80 Ellington AA, Kullo IJ, Bailey KR \& Klee GG. Antibody-based protein multiplex platforms: technical and operational challenges. Clinical Chemistry 201056 186-193. (https://doi.org/10.1373/ clinchem.2009.127514)

81 EMD Millipore. EMD Millipore's MILLIPLEX® MAP Mouse Pituitary Magnetic Bead panel Rev. 30-MAY-2017. Billerica, MA, USA: EMD Millipore Corp., 2013.

Received in final form 5 March 2018

Accepted 14 March 2018

Accepted Preprint published online 14 March 2018 http://www.endocrineconnections.org https://doi.org/10.1530/EC-18-0035

(c) 2018 The authors Published by Bioscientifica Ltd

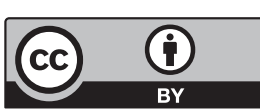

This work is licensed under a Creative Commons Attribution 4.0 International License. 